\title{
Caenorhabditis nematodes colonize ephemeral resource patches in neotropical forests.
}

Solomon A. Sloat ${ }^{1}$, Luke M. Noble ${ }^{1}$, Annalise B. Paaby ${ }^{1,2}$, Max Bernstein ${ }^{1}$, Audrey Chang ${ }^{1}$, Taniya Kaur ${ }^{1,3}$, John Yuen ${ }^{1,4}$, Sophia C. Tintori ${ }^{1}$, Jacqueline L. Jackson ${ }^{1}$, Arielle Martel ${ }^{1}$, Jose A. Salome Correa ${ }^{1}$, Lewis Stevens ${ }^{5}$, Mark Blaxter ${ }^{5}$, Matthew V. Rockman ${ }^{1}$

1. Department of Biology and Center for Genomics and Systems Biology, New York University, New York, NY 10003.

2. School of Biological Sciences, Georgia Institute of Technology, Atlanta, GA, 30332

3. Renaissance School of Medicine, Stony Brook University, Stony Brook, NY, 11974

4. Department of Molecular and Cell Biology, University of California, Berkeley, CA, 94720

5. Tree of Life, Wellcome Sanger Institute, Hinxton, CB10 1SA, UK.

Correspondence to SAS and MVR: sas958@nyu.edu and mrockman@nyu.edu

\author{
ABSTRACT \\ Factors shaping the distribution and abundance of species include life-history traits, population \\ structure, and stochastic colonization-extinction dynamics. Field studies of model species \\ groups help reveal the roles of these factors. Species of Caenorhabditis nematodes are highly \\ divergent at the sequence level but exhibit highly conserved morphological uniformity, and many \\ of these species live in sympatry on microbe-rich patches of rotten material. Here, we use field \\ experiments and large-scale opportunistic collections to investigate species composition, \\ abundance, and colonization efficiency of Caenorhabditis in two of the world's best studied \\ lowland tropical field sites: Barro Colorado Island in Panamá and La Selva in Sarapiquí, Costa \\ Rica. We observed seven species of Caenorhabditis, four of them known only from these \\ collections. While these localities contain species from many parts of the phylogeny, both \\ localities were dominated by globally distributed androdiecious species. We found that \\ Caenorhabditis were able to colonize baits accessible only by phoresy, preferring to colonize \\ baits making direct contact with the ground. We estimate founder numbers per colonization \\ event to be low.
}




\section{INTRODUCTION}

Caenorhabditis (Osche, 1952) is diverse. High sequence divergence separates even closely related sister species (Dey et al. 2012; Ren et al. 2018). Many of these species live in sympatry, yet highly conserved morphology makes it largely impossible to distinguish them without the use of molecular tools or mating tests (Sudhaus \& Kiontke 2007). The morphological uniformity among species raises questions about their long-term phenotypic stasis, species coexistence, and the niches they occupy. Previous studies of wild populations of Caenorhabditis find that they live on microbe-rich patches of decaying fruit and vegetable matter (Felix \& Duveau 2012; Felix et al. 2013; Frézal \& Félix 2015; Ferrari et al. 2017; Schulenberg \& Felix 2017; Crombie et al. 2019), the stages on which niche partitioning and interspecific competition play out. Stochastic colonization and extinction rates on these ephemeral resources are key parameters in understanding the local coexistence of species (Dubart et al. 2019).

Previous efforts to identify a Caenorhabditis substrate niche have classified several species as specialists (Kanzaki et al. 2018; Li et al. 2014; Dayi et al. 2021). The majority, however, have no obvious substrate preference. Studies of Caenorhabditis microbiomes in both laboratory (Berg, Zhou, and Shapira 2016) and the field (Dirksen et al. 2016; F. Zhang et al. 2017) suggest that animals regulate the composition of their gut flora on substrates with differing microbial composition. From these data one could hypothesize that species are specialists, occupying niches defined by what they eat. However, it is unclear which microbes are the primary food source of worms in the wild (Schulenburg \& Félix 2017). Beyond food, other factors including predators and pathogens along with non-biological sources of variation like humidity and temperature may play a role in determining where Caenorhabditis both colonize and proliferate (Félix \& Duveau 2012; Crombie et al. 2019). Field experiments have repeatedly concluded that Caenorhabditis are much more likely to be found in rotting material than in soil (Frézal \& Félix 2015; Schulenburg \& Félix 2017). Still missing is substantial evidence that Caenorhabditis preferentially colonize specific substrates like fruits or flowers. However, one field study found the degree to which a patch is rotting may influence the incidence of species found on those patches (Ferrari et al. 2017).

Equally critical to understanding Caenorhabditis adaptation to a metapopulation structure is determining modes of dispersal. Two models described by Slatkin (1977) represent the extremes of a theoretical spectrum. In the propagule pool model all colonists are derived from a single patch whereas in the migrant pool model colonizers come from the metapopulation at large. In Caenorhabditis, these models parallel hypothesized modes of dispersal, either by a phoretic host (Woodruff and Phillips 2018; Sudhaus et al. 2011; Kiontke 1997; Yoshiga et al. 2013) or by forming a semi-mobile 'seed bank' crawling towards or waiting (as specialized dauer larvae) for a fresh patch (Cutter 2015). Caenorhabditis are routinely collected from invertebrate vectors, but the contrasting modes have yet to be studied quantitatively. These contrasting modes of dispersal may have profound effects on the level of inbreeding and genetic diversity (Li et al. 2014). In addition, propagule size may contribute to the evolution of a female-biased sex ratio and the evolution of self-fertile hermaphroditism as a means of generating a population growth advantage and reproductive assurance, respectively (Theologidis et al. 2014; Cutter et al. 2019; Hamilton 1967; Lo et al. 2021).

To better understand Caenorhabditis diversity and the factors that influence it, we performed field surveys and experiments in two of the most intensively studied lowland tropical 
forests on Earth: Barro Colorado Island $(\mathrm{BCl})$, Panamá, and La Selva, Costa Rica. Barro Colorado Island lies in the center of the Panama Canal on the man-made Lake Gatún. Shortly after its formation, the island was designated a protected nature reserve and has been hosting field research for the last 100 years (Leigh 1999). Likewise, La Selva Biological Field Station in Sarapiquí, Costa Rica, has been a protected research forest for nearly 70 years (McDade et al. 1994). We focused our collection efforts on these two localities as they are relatively undisturbed by human activity and their histories of intensive research provide a rich source of information about the local ecology. One nematode metagenetic study previously found Caenorhabditis DNA in a soil and leaf litter sample at $\mathrm{BCl}$, but the species were not identified (Porazinka et al. 2010). In contrast to the majority of previous work on Caenorhabditis in the tropics, which involved transporting substrates out of country and isolating animals from nematode growth medium plates days or weeks later, we isolated and cultured all animals immediately, in the field, potentially reducing sampling biases that favor species that survive transport and grow well on Nematode Growth Medium. One other study used a combination of these approaches (Félix et al. 2013).

In total, we collected seven species of Caenorhabditis, four of them known only from these collections (including C. becei and C. panamensis, which we have described previously [Stevens et al. 2019]). Each locality was dominated by globally distributed self-fertile species. We assayed several ecological features related to patch accessibility, patch specificity, and cooccurrence of species. Using baits that vary in their accessibility we demonstrate that Caenorhabditis are able to colonize baits that are only accessible by phoresy. Further, colonization rate varied significantly with accessibility where baits making direct contact with the ground were preferentially colonized. We found that individual species tended to occur in habitat patches close to other patches of conspecifics, and we use the frequency of uncolonized patches to estimate the number of colonization events per patch. Taken together our data support current models of most Caenorhabditis species as habitat generalists whose population biology is strongly influenced by metapopulation dynamics.

\section{MATERIALS AND METHODS}

\section{Collections}

We collected nematodes on BCl in May 2012 (wet season), March 2015 (dry season), and August 2018 (wet season). Schemes for sampling varied within and among sampling sessions as described in the results. In all cases, worms were isolated from substrates and transferred to Nematode Growth Medium (NGM) plates at the $\mathrm{BCl}$ field station and identified as Caenorhabditis by morphology under a stereomicroscope. In most cases, material from the forest (e.g., rotting fruits and flowers) was placed directly onto NGM plates and Caenorhabditis worms picked to new plates to establish cultures (Barrière \& Félix 2005). These individual patches of organic material are defined as samples in our dataset and were evaluated for the presence of nematodes. For the majority of samples collected in 2018, worms were isolated by Baermann Funnel technique (Baermann 1917). Cultures of Caenorhabditis nematodes were transported to New York for species determination. Species were identified by a combination of $18 S$ and ITS2 rDNA sequencing to derive a prediction and then experimental crosses with 
isolates of known species identity to establish a biological species assignment (Félix et al. 2014; Ferrari et al. 2017; Stevens et al. 2019).

We collected nematodes at La Selva, Costa Rica, in July 2019, by Baermann Funnel. We used two methods to identify Caenorhabditis to species. Individual Caenorhabditis worms were chopped with razor blades, transferred to Whatman paper (Marek et al. 2014), and transported to New York. There the worms were identified to species by ITS2 sequencing. Separately, we established isofemale cultures on NGM plates. These plates were stored at La Selva for six months prior to their transport to New York, where surviving cultures were revived and species identified by test crosses.

Complete collection data are reported in Supplementary File 1.

\section{Sequencing and assembling the Caenorhabditis sp. 57 transcriptome}

We generated the C. sp. 57 inbred line QG3077 by 28 generations of full-sib mating from isofemale line QG3050. We generated RNA-seq mRNA transcriptome data using a pool of five mixed-stage populations of QG3077, with each population being subjected to a different condition. All worms were grown at $25^{\circ} \mathrm{C}$ on $10 \mathrm{~cm} \mathrm{NGMA} \mathrm{plates} \mathrm{(for} 1 \mathrm{~L}: 3 \mathrm{~g} \mathrm{NaCl}, 5 \mathrm{~g}$ bactopeptone, $10 \mathrm{~g}$ agar, $7 \mathrm{~g}$ agarose, $1 \mathrm{~mL}$ cholesterol $5 \mathrm{mg} / \mathrm{mL}$ in ethanol, $1 \mathrm{~mL} \mathrm{CaCl} 21 \mathrm{M}, 1 \mathrm{~mL}$ $\mathrm{MgSO}_{4} 1 \mathrm{M}, 25 \mathrm{~mL} \mathrm{KPO} 41 \mathrm{M}$ ). One population was fed with CemBio strains (Dirksen et al. 2020), and the other four were fed with E. coli OP50. The conditions for OP50 populations consisted of 1) mixed-stage, 2) starved, 3) Heat-stressed, 4) Cold-stressed. Temperature stress consisted of exposing the worms to either $35^{\circ} \mathrm{C}$ or $4{ }^{\circ} \mathrm{C}$ for 2 hours followed by a 2-hour recovery prior to RNA extraction. Total RNA was isolated using TriZol following the protocol described in Green and Sambrook (2020). The mRNA library was constructed using the Illumina Stranded mRNA Prep Ligation protocol. The library was sequenced using a NextSeq 500 MidOutput 2X150 for 300 cycles. Paired-end sequences were trimmed with Trim Galore (https://github.com/FelixKrueger/TrimGalore). Trimmed sequences were assembled into a transcriptome using Trinity (Grabherr et al. 2013) also running default parameters for paired-end reads. We then generated the longest predicted ORFs using TransDecoder (https://github.com/TransDecoder/TransDecoder) for use in phylogenetic analyses.

\section{Sequencing and assembling the Caenorhabditis sp. 24 genome}

After thawing isofemale strain QG555, the nematodes were bleached and grown on 90 $\mathrm{mm}$ NGMA plates. We harvested nematodes just after starvation and washed using M9 several times to remove $E$. coli. For genomic DNA extraction, the nematode pellets were suspended in $600 \mu \mathrm{L}$ of Cell Lysis Solution (Qiagen) with $5 \mu \mathrm{L}$ of proteinase $\mathrm{K}(20 \mu \mathrm{g} / \mu \mathrm{L})$ and incubated overnight at $56^{\circ} \mathrm{C}$ with shaking. The following day, the lysate was incubated for one hour at $37^{\circ} \mathrm{C}$ with $10 \mu \mathrm{L}$ of RNAse $\mathrm{A}(20 \mu \mathrm{g} / \mu \mathrm{L})$ and the proteins were precipitated with $200 \mu \mathrm{L}$ of protein precipitation solution (Qiagen). After centrifugation, we collected the supernatant in a clean tube and precipitated the genomic DNA using $600 \mu \mathrm{L}$ of isopropanol. The DNA pellets were washed in $70 \%$ ethanol and dried for one hour before being resuspended in $50 \mu \mathrm{L}$ of DNAse free-water. For RNA extraction, we resuspended $100 \mu \mathrm{L}$ of nematode pellet in $500 \mu \mathrm{L}$ of Trizol (5 volumes of Trizol per volume of pelleted nematodes). The Trizol suspension was frozen in liquid nitrogen and then transferred to a $37^{\circ} \mathrm{C}$ water bath to be thawed completely. This freezing/thawing process was repeated four to five times and the suspension was vortexed for $30 \mathrm{sec}$ and let rest for 30 
sec (five cycles). A total of $100 \mu \mathrm{L}$ chloroform was added and the tubes were shaken vigorously by hand for $15 \mathrm{sec}$ and incubated for 2-3 min at room temperature. After centrifugation (15 min at $13,000 \mathrm{rpm}$ and $4^{\circ} \mathrm{C}$ ), the aqueous (upper) phase containing the RNA was transferred to a new tube and precipitated with $250 \mu \mathrm{L}$ of isopropanol. The pellets were washed in $70 \%$ ethanol and dried for 15-20 min before being resuspended with 50-100 $\mathrm{LL}$ of RNAse-free water. An aliquot of each DNA and RNA preparation was run on agarose gel to check their quality and quantitated with Qubit (Thermo Scientific). Two short-insert (insert sizes of 300 and $600 \mathrm{bp}$, respectively) genomic libraries and a single short-insert (150 bp) RNA library were prepared using Illumina Nextera reagents and sequenced (125 bases, paired-end) on an Illumina HiSeq 4000 at Edinburgh Genomics (Edinburgh, UK). All raw data have been deposited in the relevant International Nucleotide Sequence Database Collaboration (INSDC) databases.

We performed quality control of our genomic and transcriptomic read sets using FastQC (v0.11.9; Andrews and Others 2010) and used fastp (0.20.1; Chen et al. 2018; --length_required $50)$ to remove low-quality bases and Illumina adapter sequence. We generated a preliminary genome assembly using SPAdes (v3.14.1; Bankevich et al. 2012; --only-assembler --isolate -k $21,33,55,77)$ and identified the likely taxonomic origin of each contig by searching against the NCBI nucleotide (nt) database using BLASTN (2.10.1+; Camacho et al. 2009; -task megablast max_target_seqs 1 -max_hsps 1 -evalue 1e-25) or by searching against UniProt Reference Proteomes database using Diamond BLAST (2.0.4; Buchfink, Xie, and Huson 2015; --max-targetseqs 1 --sensitive --evalue 1e-25). We also mapped the genomic reads to the genome assembly using bwa mem (0.7.17-r1188; Li 2013). We provided the assembly, the BAM file, and the BLAST and Diamond files to blobtools (1.1.1; Laetsch and Blaxter 2017) to generate taxon-annotated, GC-coverage plots, which we used to identify contaminant contigs. Any read pair that mapped to the contaminant contigs was discarded. Using this filtered read set, we generated a final assembly using SPAdes (--isolate $-k 21,33,55,77,99)$. We also generated a transcriptome assembly using Trinity (Trinity-v2.8.5; Haas et al. 2013), which we then used to scaffold the genome assembly using SCUBAT2 (available at https://github.com/GDKO/SCUBAT2). We used numerical metrics and BUSCO (v4.1.4; Simão et al. 2015; -I nematoda_odb10 -m genome) to assess assembly quality and biological completeness, respectively. Prior to gene prediction, we generated a species-specific repeat library using RepeatModeler (2.0.1; A. Smit and Hubley 2010; -engine ncbi), and combined this library with known Rhabditid repeats from RepBase (Jurka et al. 2005). This repeat library was then used to soft-mask the genome using RepeatMasker (open-4.0.9; A. F. A. Smit, Hubley, and Green 1996; -xsmall). We predicted genes in the genome by aligning trimmed transcriptomic data to the genome using STAR (2.7.3a; Dobin et al. 2013; -twopassMode Basic) and providing the resulting BAM file to BRAKER2 for gene prediction (2.1.5; Brůna et al. 2021; --softmasking). We used BUSCO (-I nematoda_odb10 -m proteins) to assess gene set completeness.

\section{Phylogenetic analysis}

We identified a set of orthologous proteins by running BUSCO (Seppey et al. 2019) using the nematode_odb10 dataset on each nematode genome found in Table S1. Multisequence fasta files for each ortholog were extracted using busco2fasta (https://github.com//stevens17/busco2fasta) with the setting -p 0.8, meaning each ortholog was required to be in $80 \%$ or 28 of the 36 species. Orthologous sequences were then aligned with 
MAFFT (Katoh and Standley 2013) and ML gene trees estimated using IQ-TREE (Nguyen et al. 2015), both on default settings. Newick trees were concatenated into a single file and a species tree was estimated using ASTRAL-III (C. Zhang et al. 2018), which uses a coalescent framework. We also generated a species tree using a supermatrix of all concatenated orthologs. To generate the supermatrix we used TrimAl (Capella-Gutiérrez et al. 2009) to remove poorly aligned regions using the settings -gt 0.8 -st 0.001 -resoverlap 0.75 -seqoverlap 80 . Sequences were subsequently concatenated using catfasta2phyml (https://github.com/nylander/catfasta2phyml). A tree was then inferred with IQ-TREE using the LG substitution model (Le and Gascuel 2008), modeling the rate variation among sites using a Discrete Gamma model (Yang 1994) with 4 categories. Support was estimated using 1000 ultrafast bootstrap replicates (Hoang et al. 2018). We then estimated ASTRAL-III tree branch lengths in units of replacements per site rather than coalescent units using IQ-TREE with the same parameters as the supermatrix analysis while fixing the tree by the output of the ASTRALIII analysis using the -te setting. All newick trees were visualized using the ITOL web browser (Letunic and Bork 2019).

\section{RESULTS}

\section{The Caenorhabditis faunas of $\mathrm{BCl}$ and La Selva}

We recovered Caenorhabditis nematodes from 225 samples collected on $\mathrm{BCl}$ (Figure 1; Supplementary File 1). Additional samples did not contain Caenorhabditis or were damaged during processing and shipping. The Caenorhabditis isolates derive from opportunistic sampling of rotten fruits, flowers, mushrooms, and leaf litter in 2012 and 2018, from systematic sampling of Gustavia superba flowers in 2012, and from several classes of experimental baits in 2015. By DNA barcode sequencing and laboratory mating tests (Sudhaus et al. 2011; Félix et al. 2014), we assigned the Caenorhabditis isolates to six different species, three of which are currently known only from our collections on BCl. These are C. becei Stevens 2019, C. panamensis Stevens 2019, and C. sp. 57. The number of samples yielding each species is shown in Table 1. In total, the 225 samples yielded 260 species observations, as many samples contained multiple Caenorhabditis species.

\section{TABLE 1}

$\begin{array}{llll}\text { Species } & \text { Total Positive Samples } & 2018 \text { Survey } & \text { Range } \\ \text { C. briggsae } & 152 & 26 & \text { cosmopolitan } \\ \text { C. tropicalis } & 43 & 15 & \text { pantropical } \\ \text { C. panamensis } & 30 & 11 & \text { endemic } \\ \text { C. becei } & 25 & 10 & \text { endemic } \\ \text { C. sp. } 24 & 8 & 6 & \text { neotropical } \\ \text { C. } \mathrm{sp} .57 & 2 & 1 & \text { endemic }\end{array}$

To assess the completeness of our survey, we used rarefaction of the chao2 incidencebased estimator (Hsieh, Ma, and Chao 2016; Chao et al. 2014), which generated an estimated species richness of $6 \pm 0.34(95 \% \mathrm{Cl})$ (Figure 1). These data suggest that we have recovered the maximum number of species at $\mathrm{BCl}$, conditional on our sampling strategy. The two most 
abundant species, C. briggsae and C. tropicalis, are androdioecious (males and self-fertile hermaphrodites), and their geographic distributions are cosmopolitan and pantropical, respectively. The other species are gonochoristic (males and females). One of these species, $C$. sp. 24, has also been found in French Guiana (Ferrari et al. 2017), Mexico, and Southern California (personal observation and Félix 2021).

We successfully recovered Caenorhabditis nematodes from 77 samples at La Selva, Costa Rica (Figure 1; Supplementary File 1). These derive from opportunistic sampling of rotten fruits, flowers, mushrooms, and litter in 2019. These samples yielded only 3 different species, one of which is known only from our collections at La Selva (C. sp. 60). La Selva differed from $\mathrm{BCl}$ in that $C$. tropicalis was most prevalent (present in 55 samples), followed by C. briggsae (32 samples). Gonochoristic C. sp. 60 was isolated from a single substrate, which contained an estimated thousands of individuals. Rarefaction of the chao2 incidence-based estimator generates a species richness of $3 \pm 0.48(95 \% \mathrm{Cl})$ (Figure 1). This suggests that the lower number of observed species at La Selva is not due to inadequate sampling given our sampling strategy. We measured substrate temperature for 22 samples that contained Caenorhabditis; these ranged from 24.1 to $28.4{ }^{\circ} \mathrm{C}$, with each species averaging $26{ }^{\circ} \mathrm{C}$ (Supplementary File 1).

A

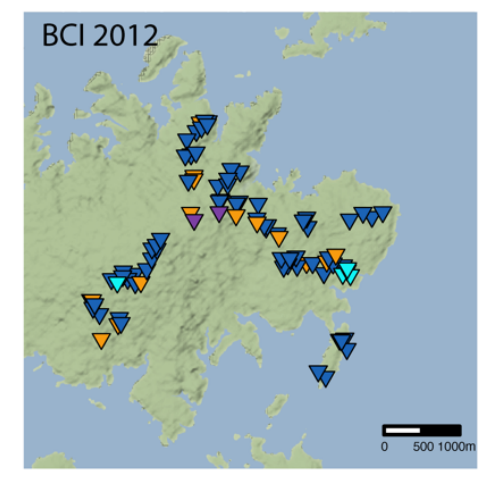

D

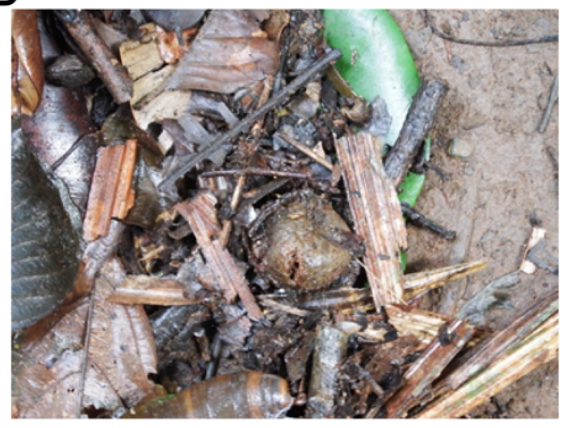

B

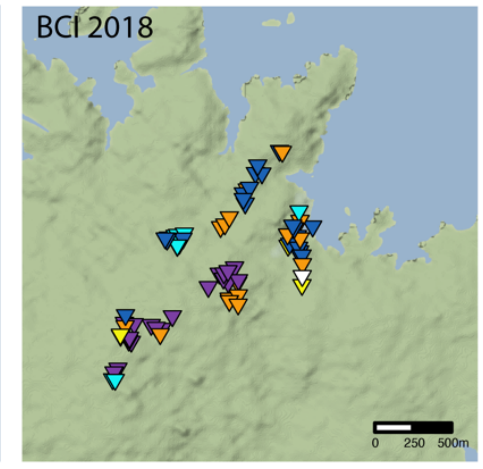

$E$
C

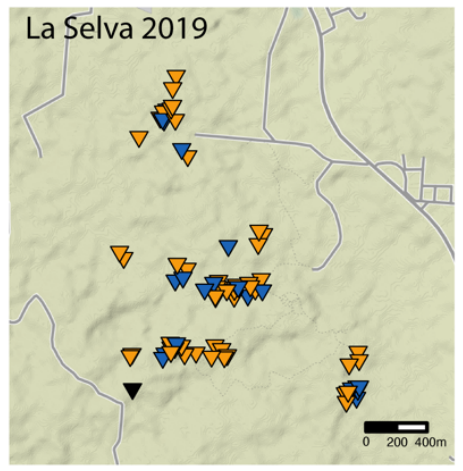

Species

$\nabla$ c. becei

$\nabla$ c. briggsae

$\nabla$ c. panamensis

$\nabla$ C.sp. 24

$\nabla$ c.sp. 57

$\nabla$ C. tropicalis
C. sp. 60

Figure 1. Caenorhabditis were collected at two localities: Barro Colorado Island, Panamá and La Selva in Sarapiquí, Costa Rica. (A-C) Distribution of species collected from opportunistic sampling from each locality by year. Each marker represents a patch positive for that species. Patches may be plotted multiple times if species co-occurred on the same patch. Patches are jittered to prevent overpotting. (D) A fig substrate from which C. sp. 57 was isolated. (E-F) Rarefaction curve of the chao2 incidence-based estimator for both localities. The solid line represents the predicted species richness the dotted line represents an extrapolation of species richness. The grey area is the $95 \%$ confidence interval. 
To understand the phylogenetic positions of the undescribed species, we sequenced and assembled a transcriptome for $C$. sp. 57 and a genome of $C$. sp. 24. Using these assemblies and the assemblies of 34 additional Caenorhabditis species, we identified 1931 single-copy orthologs that were represented in at least 28 of the 36 species. We estimated the Caenorhabditis phylogeny using two approaches. First, we used a coalescent-based approach with individual gene trees as input. Second, we used a maximum likelihood approach using a concatenated alignment of all orthologues as input. The resulting phylogenies (Figure 2) exhibit largely congruent topologies that are consistent with previous analyses (Stevens 2019), differing only in the position of C. virilis. C. sp. 24 is closely related to C. quiockensis (Stevens 2019) within the Angaria group of spiral-mating species (Sudhaus et al. 2011). C. sp. 57 is most closely related to C. monodelphis (Slos \& Sudhaus 2017) and C. auriculariae (Tsuda \& Futai

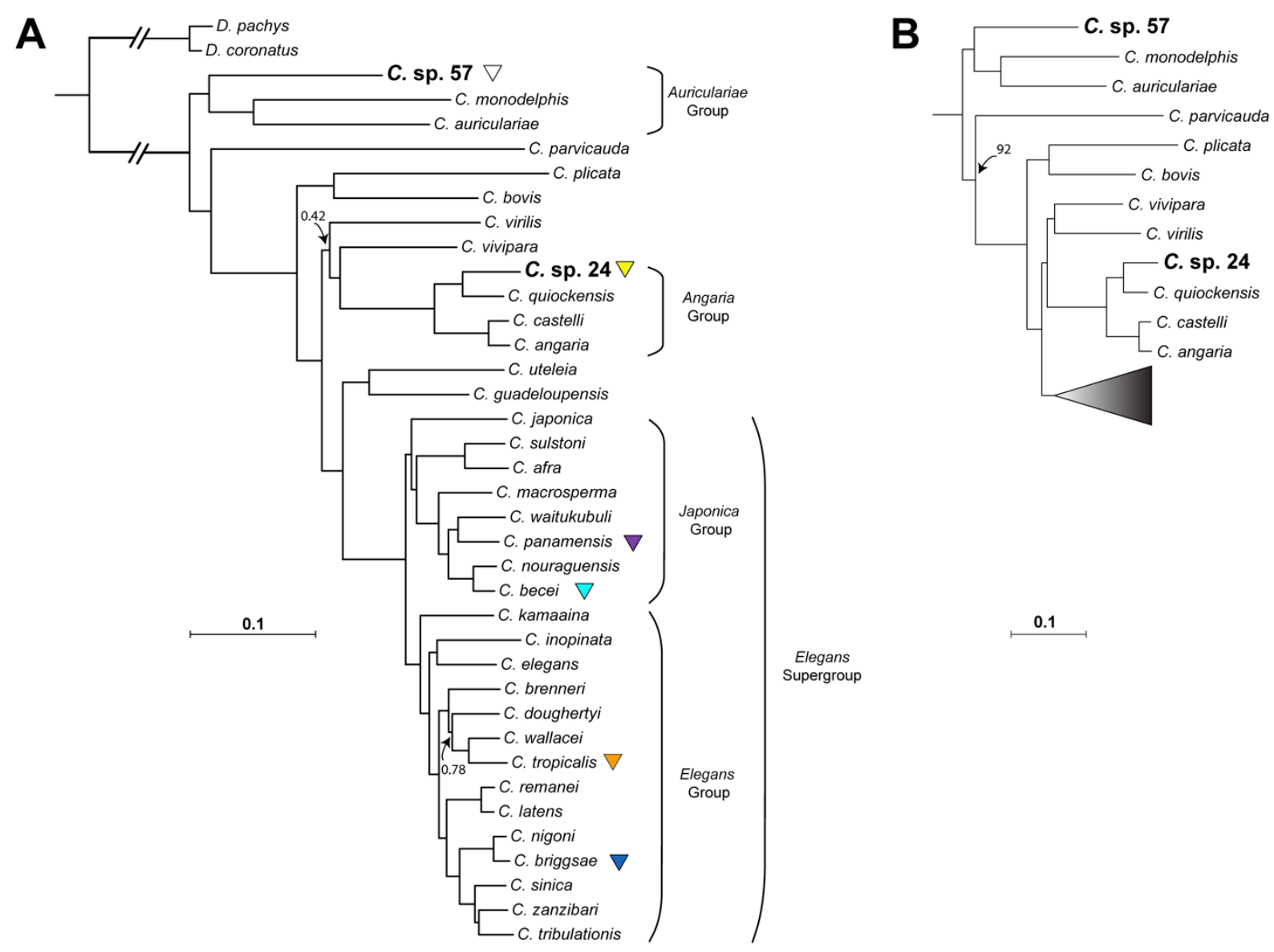

Figure 2. Phylogeny of 36 Caenorhabditis species with $D$. coronatus and $D$. pachys forming an outgroup based on 1,931 single-copy orthologs each shared between $80 \%$ of the species. (A) Phylogeny inferred using a coalescent approach that takes gene trees as input (substitution models for each gene tree selected automatically). Branch lengths in substitutions per site were estimated using the LG substitution model with gamma-distributed rate variation among sites $(L G+\Gamma)$ while fixing the phylogeny to the coalescent tree topology. Species incorporated into the phylogeny for the first time are bolded. Posterior probabilities are 1.0 unless noted. (B) Alternative topology using a supermatrix approach that uses concatenated alignments of all orthologs as input under a $L G+\Gamma$ model. Bootstrap support is 100 unless noted. 
1999), which together form the sister group to all other Caenorhabditis. From the ITS2 sequence alone C. sp. 60 is sister to C. macrosperma within the Japonica group (NCBI Accession: OL960095). Overall, the species found at BCl and La Selva span the Caenorhabditis phylogeny. The two selfers, $C$. briggsae and C. tropicalis, are the sole representatives of the Elegans group, while three species (C. becei, C. panamensis, C. sp. 60) are members of a neotropical-endemic clade within the Japonica group.

\section{Substrate specificities}

To minimize variation due to differences in sampling technique, we limited our substrate analysis to a dataset of 177 samples collected and processed by a single investigator in August 2018. These samples included a range of rotten fruits, flowers, stems, fungi, and leaf litter. Overall, $94 \%$ of the samples yielded nematodes, and 32\% (57/177) yielded Caenorhabditis. Some samples contained multiple Caenorhabditis species, totaling 69 species observations (Table 1).

The species abundance ranks match those from the remaining pool of all observations, though the proportions are different, with C. briggsae less overwhelmingly dominant. Each of the four most common species was collected from multiple types of fruit and flower. Classifications of the substrates, at high levels (fruit vs other) or lower (e.g., Spondias mombin fruit vs. fig), revealed no significant association between Caenorhabditis generally or any species specifically and any substrate. Acknowledging the very limited statistical power for most of these tests, we interpret this as evidence that the common species are substrate generalists, colonizing and proliferating in any available habitat patch.

\section{The spatial patterning of patch occupancy}

To understand the spatial patterning of Caenorhabditis among habitat patches, we performed hierarchical spatial sampling of a single substrate type, rotten flowers of Gustavia superba, in May 2012. We selected four G. superba trees spread across the island, and at each we established three well separated $1 \mathrm{~m}^{2}$ quadrats. Within each quadrat, we sampled four rotten flowers, each at least $10 \mathrm{~cm}$ apart. From each flower that yielded Caenorhabditis, we established isofemale or isohermaphrodite lines from four or more randomly selected worms from each flower. At one tree only two quadrats were sampled. In total this sampling scheme involved 44 samples of $G$. superba flowers.

Thirty-six of 44 G. superba flowers (82\%) contained Caenorhabditis. C. briggsae was present in every Caenorhabditis-positive quadrat at every site, while the other species exhibited strongly patchy distributions over scales of meters (Figure 3). For example, $C$. becei was present in all four flowers in one quadrat at Plot DFT but absent from the flowers in the other two quadrats there. Similarly, $C$. tropicalis was present in three of four flowers in one quadrat at Plot DT but absent from the other two quadrats a few meters away. This patchiness is manifest at larger scales as well: $C$. panamensis was present in all three quadrats at Plot StLT but absent from the other three plots.

C. briggsae was present in 29 of the 44 G. superba flowers (66\%). This allows a crude estimate of the number of flowers colonized by $C$. briggsae multiple times. If $C$. briggsae is present ubiquitously and patch colonization is a Poisson process, the absence of $C$. briggsae from $34 \%$ of flowers implies a Poisson-distributed number of colonizations per patch with mean 
1.08 , with $29 \%$ of flowers colonized by C. briggsae more than once. Thus $\sim 44 \%$ of the flowers that contained $C$. briggsae $(0.29 / 0.66)$ are expected to have had multiple colonizations.

There is no evidence that the presence of one species affects the probability of observing a second species within a sample. For example, C. briggsae and C. tropicalis are present in $66 \%$ and $9 \%$ of the 44 samples; the expected co-occurrence under independence is $2.6 / 44$ and we observe co-occurrence of $3 / 44$ samples.
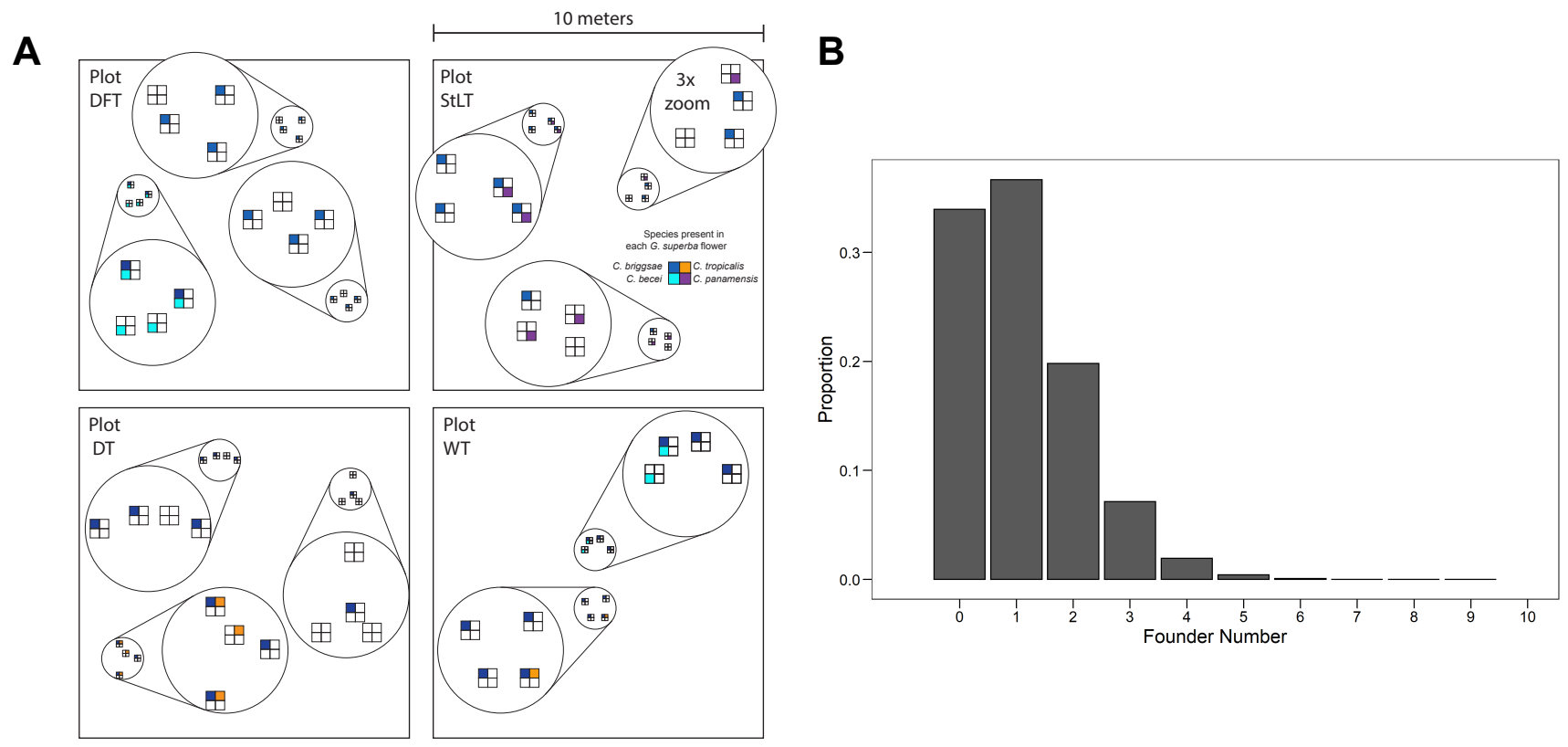

Figure 3. Species are patchily distributed among rotting Gustavia superba flowers. (A) 10x10 meter plots were systematically sampled at each of four focal trees. At each plot, four flowers were collected from two or three 1-meter quadrats. Each box represents a flower, each color represents the species present on that flower $(B)$ The distribution of $C$. briggsae colonization events per flower under a simple Poisson model (mean=1.08).

\section{Colonization patterns among classes of bait}

To test how substrate type and accessibility affect rates of colonization by Caenorhabditis, we set up arrays consisting of several bait types. At each of seven sites on $\mathrm{BCl}$, we set up a 7-by-7-meter field site with five arrays of baits (four in the corners, one in the center). Each bait array consisted of six agar baits, each bait of a different type (Figure 4), arranged $3 \times 2$ with $30 \mathrm{~cm}$ spacing between the 6 -cm diameter baits. Our experiment as a whole therefore included 210 baits in total. Baits were placed on March 242015 and were collected on March 27 2015, at which time a sample of the bait was placed on a seeded plate and the plate was monitored for nematodes twice daily for four days. Twenty-nine of the 210 baits were absent at the time of collection (in cases we observed, eaten by ants and beetles), leaving data for 181 baits for analysis. From each bait that yielded nematodes, we identified Caenorhabditis by morphology and established lines. From each Caenorhabditis-positive sample we determined the species for at least one line by mating tests.

From 181 baits recovered after three days in the forest, we found $56(31 \%)$ colonized by nematodes, including 17 (9\%) colonized by Caenorhabditis (15 C. briggsae and 3 C. tropicalis, 
including one bait with both species). Colonization rates varied significantly by bait type, for worms overall ( $p<10^{-12}$; analysis of deviance from logistic regression), for Caenorhabditis generally $(p=0.001)$, and for $C$. briggsae specifically $\left(p<10^{-4}\right)$. Caenorhabditis showed a baittype distribution that does not differ significantly from the distribution of baits colonized only by non-Caenorhabditis nematodes (Fisher's exact test, $p=0.92$ ), though the power of this test is limited by the small size of the data set. Another way to state this is that the probability of Caenorhabditis in a bait type is correlated with the probability of only non-Caenorhabditis worms in a bait type $\left(r^{2}=0.98, p<0.001\right)$.

A

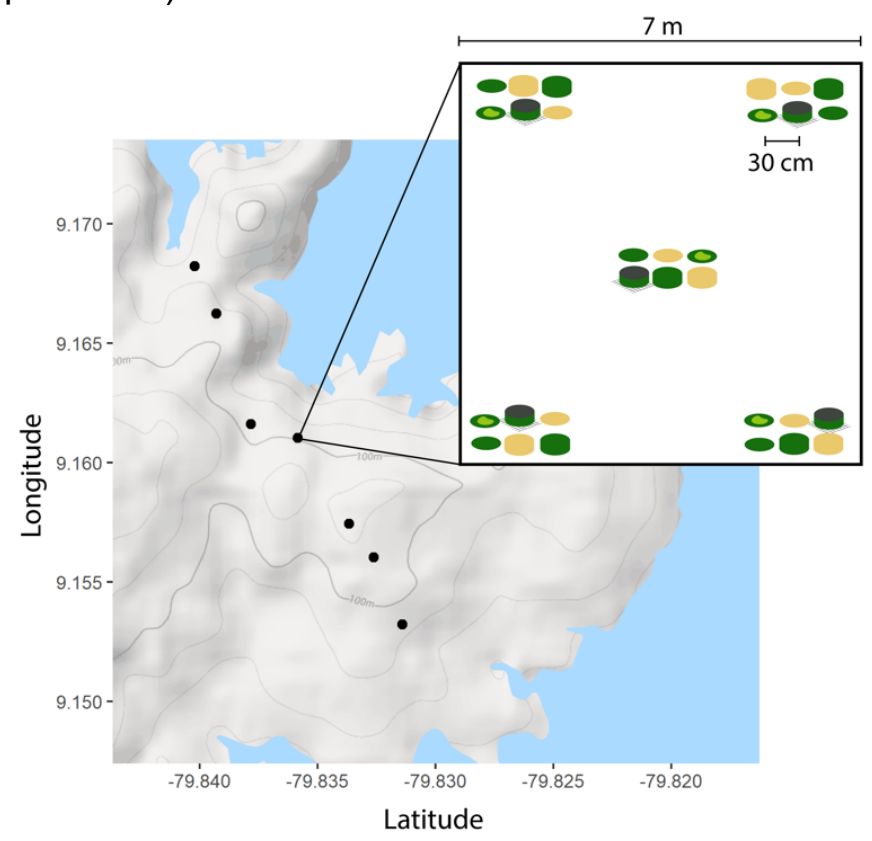

B

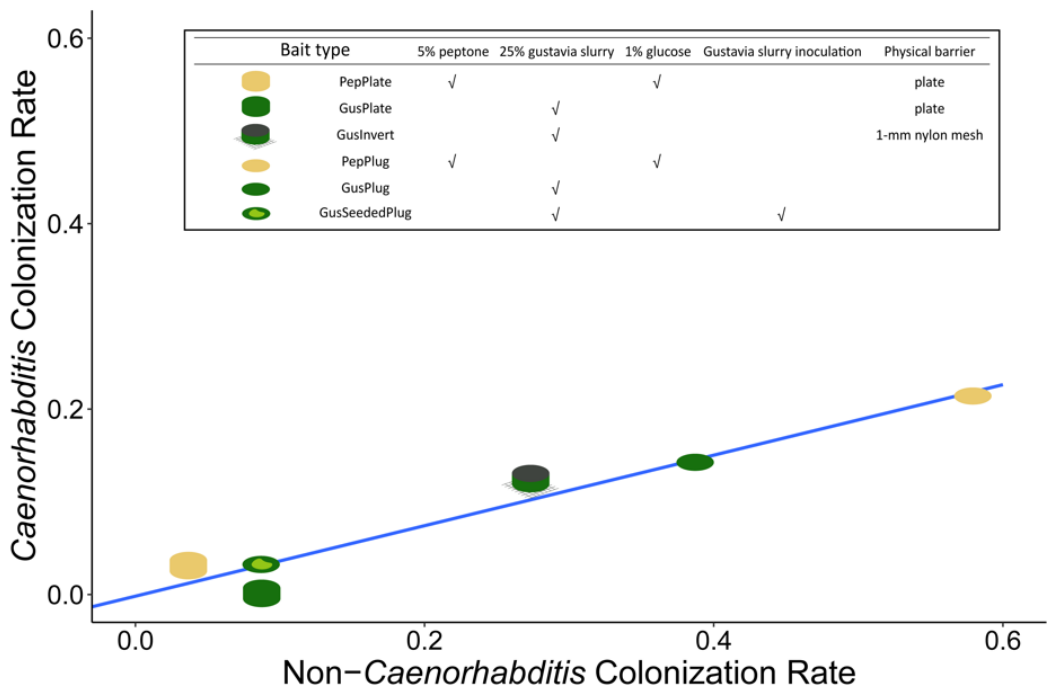

Figure 4. Colonization rates vary in response to bait composition and accessibility. (A) Baits were set up at each of seven sites across $\mathrm{BCl}$. Each site consisted of 30 baits arranged in groups of six in the corners and center of each site. (B) Six types of agar bait differing in recipe and presence of barriers to access, showed different rates of colonization by nematodes Blue line is linear regression of Caenorhabditis vs. Non-Caenorhabditis colonization rates across bait types. Table inset contains the composition and accessibility of each bait type. 
The worms preferentially colonized plug baits, which make direct contact with the ground, over plate baits, which are isolated from the ground by plastic. In both plates and plugs, the worms preferentially colonized those with peptone enrichments over those with heatdefaunated Gustavia superba flower slurry. And among Gustavia plugs, they preferentially colonized those that were not supplemented with raw Gustavia slurry.

\section{Test of colonization by phoresy}

We used size-selective exclosures to determine whether colonization requires phoresy on animals of particular sizes. In 2015, we set up arrays of 24 baits in a $6 \times 4$ grid, with 1 meter spacing between samples, at each of six locations spread across $\mathrm{BCl}$. The baits consisted of Gustavia superba flower slurry, made by homogenizing flowers and water in a kitchen blender and then heating the mixture to defaunate it. Each array of 24 samples included 4 replicates of 6 different treatments. One treatment consisted of slurry deposited directly onto the forest floor. For the other five treatments, the slurry was placed into a plastic cup and access to the slurry was restricted by the nature of the cup lid. The lids had a circular opening with $3.1 \mathrm{~cm}$ diameter, which was either totally open or covered with a nylon mesh to restrict access by animals larger than the mesh size. The mesh openings restricted passage to animals smaller than $4 \mathrm{~mm}, 1$ $\mathrm{mm}, 0.064 \mathrm{~mm}$, or $0.01 \mathrm{~mm}$.

After 5 days in the field, we collected the slurry samples and transferred a small volume (approximately $1 \mathrm{~cm}^{3}$ ) to NGM plates. If worms emerged, we attempted to establish cultures. Surviving cultures were cryopreserved in New York, and species were identified by sequencing and mating tests. One bait was lost, and of the 143 baits that we recovered, we found nematodes in 30, including three species of Caenorhabditis and at least ten additional species (Figure 5; Supplementary File 1). Because some baits were colonized by multiple species, we count 34 species observations overall.

C. briggsae and $C$. tropicalis both colonized baits inside plastic cups, demonstrating that these animals can colonize new substrates by phoresy on other animals. Conversely, Oscheius tipulae, which colonized seven baits, only colonized baits that were accessible directly from the soil or leaf litter. We observed substantial heterogeneity among the plots (Figure 4). Bait accessibility significantly affected colonization rates by nematodes generally $\left(p=5.4 \times 10^{-8}\right.$; analysis of deviance from logistic regression) and by Caenorhabditis specifically $(p=0.007)$. These analyses treat the bait accessibility as a continuous variable, but analysis with accessibility as unordered levels of a factor yields congruent results. Caenorhabditis colonized only the three most accessible classes of bait, suggest that their phoretic hosts were unable or disinclined to pass through mesh with pores of a millimeter or smaller. 
Donato

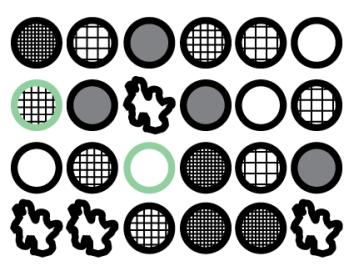

Lutz

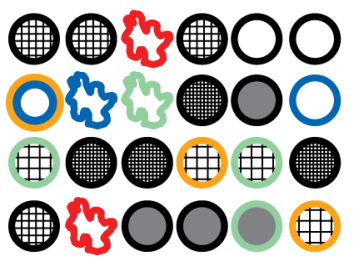

Barbour

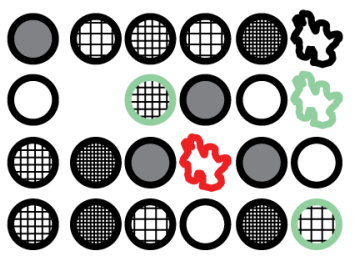

Shannon

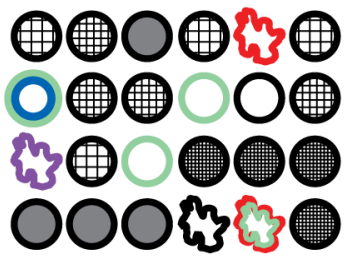

Van Tyne

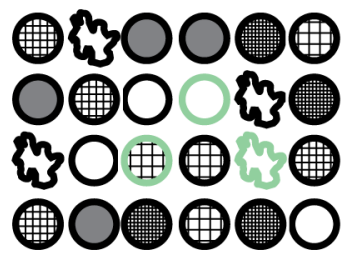

Fausto

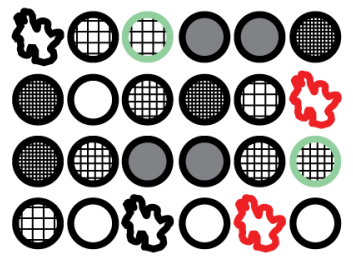

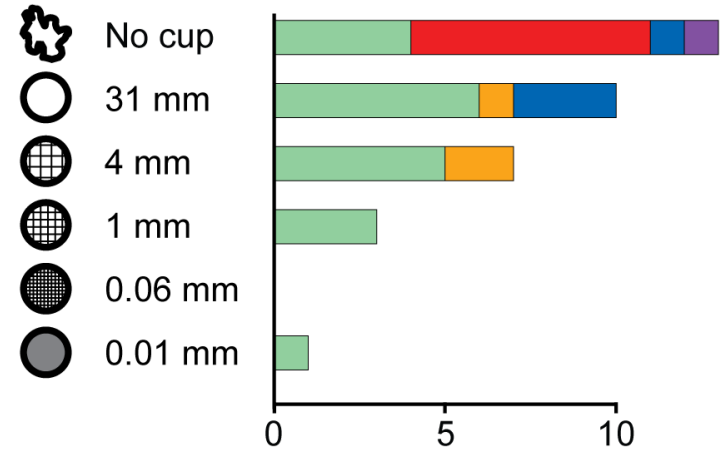

Not colonized

C. briggsae

C. tropicalis

C. panamensis

Oscheius tipulae

other nematode

Figure 5. Nematodes colonized 30 baits across six experimental plots, each containing a randomized grid of 4 replicates of each of 6 types of bait differing only in accessibility. Accessibility ranged from no barrier to being accessible via $0.01 \mathrm{~mm}$ pores. Colonization varied significantly by bait accessibility. $C$. tropicalis and $C$. briggsae both colonized baits isolated from the environment and accessible only by phoresy while $O$. tipulae was only found to colonize baits making direct contact with the ground.

\section{DISCUSSION}

Over the past twenty years an increasing community effort to connect the rigorously studied genetic model of Caenorhabditis to its natural environment has been fruitful. The catalogue of Caenorhabditis species and wild isolates has increased dramatically and along with it the ability to apply population, quantitative, and comparative genomic methods (Stevens et al. 2019; Cook et al. 2017). Despite these advances, a well-supported model of Caenorhabditis population biology is still being formulated. Here, we present a deep sampling of Caenorhabditis natural diversity in two of the most extensively studied neotropical field sites, along with a collection of experiments aimed at understanding Caenorhabditis in relation to their 
local metapopulation structure. In total we collected seven species, four of which are only found in these collections (BCl: C. becei, C. panamensis, and C. sp. 57; La Selva: $C$ sp. 60). We estimate that we recovered the total number of species in both field sites accessible to our sampling scheme, which is limited by various factors like time of year, selection of visibly rotting material, nematode isolation method, and proximity of sampling localities to trails. Different sampling schemes would potentially yield different results.

Species from four major clades of Caenorhabditis are found in these forests, including representatives of the Elegans and Japonica groups, the spiral-mating Angaria group, and the Auriculariae group, which is distantly related to most Caenorhabditis. Our findings comport with biogeographic hypotheses about the history of Caenorhabditis diversity. In particular, we find three species that are part of a neotropical-endemic clade within the Japonica group. Species in this group can be locally abundant in neotropical forests, but their geographic ranges appear to be quite narrow. Each species is known only from a single region, with no overlap among the species in this group found at La Selva, BCl, French Guiana, or Dominica (Stevens et al. 2019; Félix 2021). Most parts of the neotropics have not yet been surveyed for Caenorhabditis, and we infer that many Japonica-group species remain to be discovered there. Conversely, Elegans group species are represented exclusively by two widely distributed androdioecious species. Endemic gonochoristic Elegans-group species, which are quite numerous in east Asia and Australia, appear to be absent from the neotropics.

Common species at $\mathrm{BCl}$ appear to be substrate generalists. Rotten Gustavia superba flowers were often occupied by Caenorhabditis. We hypothesized that a specific microbial environment on the substrate was preferred by the worms. Our bait preference data suggest that this microbial environment requires conditions that we did not successfully replicate with fresh flower slurry. Caenorhabditis preferred baits supplemented with the general microbial growth medium peptone over the Gustavia slurry. Moreover, we found that nematode colonization rate was significantly correlated with Caenorhabditis colonization rate, suggesting that these worm communities are substrate generalists. This might also suggest that absolute quantity of microbial food is an important factor in determining where Caenorhabditis both colonize and proliferate. This conclusion is consistent with our opportunistic sampling data which found no associations between any substrate type and incidence of Caenorhabditis. Few field studies have looked at substrate preference specifically. Ferrari et al. (2017) found the incidence of Caenorhabditis on fresh fruit (citrus) baits to be enriched when compared to nonCaenorhabditis nematodes, while Crombie et al. (2019) concluded that they observed no substrate specificity between Caenorhabditis species and their opportunistically sampled substrates. Future studies would best be served by measuring the response of Caenorhabditis incidence to a larger variety of substrate baits and their microbial composition as well as the absolute quantity of microbes on those baits in order to delineate these factors.

Our data supports current models of Caenorhabditis modes of dispersal through the use of phoresy in colonization. In our exclosure experiment, Caenorhabditis colonized baits that were directly accessible from the ground, isolated from the ground in a cup, and isolated in a cup and further blocked by mesh with openings of $4 \mathrm{~mm}$ or greater. Baits isolated by mesh with openings of 1-mm or smaller were not colonized. In contrast, Oscheius tipulae only colonized baits making direct contact with the ground (although there is limited evidence for phoresy in $O$. tipulae (De Luca et al. 2019)). One hypothesis is that phoretic vectors are colonizing fresh fruit 
and flower substrates while they are still on the plant, as is the case in some specialist species like C. inopinata (Kanzaki et al. 2018). Our data confirm that phoretic vectors are mediating the colonization of substrates on the ground, leaving the possibility that animals are also colonizing substrates in trees for future testing.

Species were unevenly distributed over time and geography. There were year-to-year changes in the species collected at various localities around $\mathrm{BCl}$. For example, in 2012 collections at tree DFT yielded C. briggsae and C. becei, but in 2015 collections at that same tree yielded only $C$. tropicalis. One model is that habitat patches are colonized randomly from the local species pool, as suggested by the patchy species distribution of $G$. superba flower occupancy. An alternative is that species differences among years illustrate ecological succession at larger scales than the level of an individual substrate and its lifespan. Felix \& Duveau (2012) more systematically describe a seasonal succession in the abundance of $C$. briggsae and $C$. elegans in a French orchard, paralleling their finding that $C$. briggsae outcompetes $C$. elegans at higher temperatures in the lab. Future analyses of neotropical localities over time will better reveal the spatiotemporal dynamics of the species that coexist there.

Species in our spatial sampling data set appeared to differ in their distributions across sampling sites and quadrats. C. briggsae was present in every quadrat at every focal tree sampling site while other species had a patchier distribution over a scale of meters and at scales between focal tree sampling sites. These patterns could indicate differences in colonization efficiency and differences in the scale of dispersal between species which might be picked up by a larger dataset. Under the assumption that animals colonize patches independently and randomly, we estimated that about $44 \%$ of patches occupied by C. briggsae had multiple colonizations. Richaud et al. (2018) modeled C. elegans founder number using a Poisson distribution given the proportion of genotypes they observed at a given distance between two patches. They varied how they modeled local haplotype frequencies to account for the unknown proportions in the source population and came to a mean number of 3-10 individuals. Our estimate adds growing support to the hypothesis that founder numbers are low across Caenorhabditis and that their population biology is affected by living in an ephemeral metapopulation structure. In general, more detailed investigations into modes of dispersal will reveal a more complete model. These might include characterization of the phoretic vectors employed by Caenorhabditis species and genetic analysis of field-collected individuals at fine spatial and temporal scales. Using these data, one could construct a dispersion kernel to understand dynamics and distance of colonization and estimate founder number while minimizing assumptions. Understanding modes of dispersal is crucial to understanding patterns of diversity, inbreeding, and selective pressures that metapopulation structure imposes on traits like selfing and sex ratio.

Our data join with comparable field studies in tropical lowland sites in French Guiana and Hawaii to suggest that androdioecious species not only have larger global ranges than dioecious relatives but are also locally dominant (Table 2). Our collection efforts identify $C$. briggsae as the predominant species at $\mathrm{BCl}$ followed by $C$. tropicalis, as in lowland Hawaii (Crombie et al. 2019). At La Selva C. tropicalis is the most abundant with the sole dioecious isolate being $C$. sp. 60 . The largest contrast is Nouragues, French Guiana, where $C$. tropicalis predominates among the androdioecious species but the gonochoristic $C$. nouraguensis is the 
most abundant overall (Ferrari et al. 2017). Taken together, this suggests that the hypothesized benefits of self-fertile hermaphroditism, including reproductive assurance, population growth advantages, and resistance to Medea elements (Cutter et al. 2019; Noble et al. 2021), are adaptive at multiple spatial scales. While the molecular details of transitions to self-fertile hermaphrodism are well understood (Hill et al. 2006; Baldi et al. 2009; Woodruff et al. 2010; Wei et al. 2014), more work needs to be done to understand the factors which drive the evolution of the transition and which maintain dioecious and androdioecious species in sympatry.

\section{TABLE 2}

$\begin{array}{llcll} & \mathrm{BCl} & \text { Hawaii lowlands } & \text { Nouragues } & \text { La Selva } \\ \text { C. astrocarya } & 0 & 0 & 16 & 0 \\ \text { C. becei } & 25 & 0 & 0 & 0 \\ \text { C. brenneri } & 0 & 0 & 3 & 0 \\ \text { C. briggsae } & 152 & 88 & 37 & 32 \\ \text { C. castelli } & 0 & 0 & 1 & 0 \\ \text { C. dolens } & 0 & 0 & 1 & 0 \\ \text { C. kamaaina } & 0 & 2 & 0 & 0 \\ \text { C. macrosperma } & 0 & 0 & 9 & 0 \\ \text { C. nouraguensis } & 0 & 0 & 219 & 0 \\ \text { C. oiwi } & 0 & 12 & 0 & 0 \\ \text { C. panamensis } & 30 & 0 & 0 & 0 \\ \text { C. tropicalis } & 43 & 13 & 178 & 55 \\ \text { C. sp. } 24 & 8 & 0 & 11 & 0 \\ \text { C. sp. } 57 & 2 & 0 & 0 & 0 \\ \text { C. } 5 p .60 & 0 & 0 & 0 & 1\end{array}$

Hawaii Lowlands data are as reported in Crombie et al. (2019), including only samples collected in 2017 from elevations below $500 \mathrm{~m}$. Nouragues data are as reported in Ferrari et al. (2017), representing the count of samples containing each species summed across collections in 2013, 2014, and 2015.

\section{CONCLUSIONS}

Deep sampling of two neotropical sites yielded seven species, four of which are found only in these collections. These collections add growing evidence that self-fertile species not only have larger ranges but are locally dominant in the neotropics. Field experiments support current models of Caenorhabditis ecology in which most species are substrate generalists whose population biology is tightly linked to a metapopulation structure. Dauer animals travel from one ephemeral resource to the next on phoretic vectors founding a new population from only a small handful of individuals. These experiments help to inform future work which could more systematically build a model of Caenorhabditis metapopulation dynamics which includes species co-occurrence and competition, dispersal dynamics, founding numbers, and the effects of substrate variation and quality. 


\section{Data and code availability}

Raw sequencing data and transcriptome assembly for $C$. sp. 24 have been archived under the NCBI study accession PRJEB48807. Raw sequencing data and genome assembly and annotation files for $C$. sp. 57 have been archived under the ENA study accession PRJNA789856.

\section{Supplementary Table: Phylogenetic Data}

TABLE_S1.xIsx contains the list of all species and accessions to genomic/transcriptomic data used in the phylogenetic analysis.

\section{Supplementary File: Collection Data}

SupplementaryFile1.xlsx contains all of the collection data reported in the manuscript. Data are provided in a series of sheets corresponding to specific analyses and results, as follows:

BCI.SamplesBySubstrateAllYears: Each of the 225 rows records one substrate sample that yielded Caenorhabditis nematodes. The species found in each sample are indicated by $1 \mathrm{~s}$ in the relevant species columns.

BCI.Opportunistic2012: Each row represents an isohermaphrodite or isofemale line established from opportunistic collections in 2012.

BCI.Spatial2012: Each row represents an isohermaphrodite or isofemale line established from heirarchical spatial sampling of Gustavia superba flowers in quadrats around focal trees in 2012.

BCI.Exclosures2015: Each row represents a single bait from one of the 24 baits set out at each of six locations in 2015.

BCI.Exclusures2015Key: This sheet provides a key to the columns of the BCl.Exclusures 2015 sheet, including descriptions of the exclosure types, coordinates of the exclosures, and a summary of the species representation in each type of exclosure.

BCl.AgarBaits2015: Each row represents a single bait from one of the 30 set out at each of seven locations in 2015.

BCl.AgarBaits2015Key: This sheet provides a key to the columns of the BCl.AgarBaits2015 sheet, including descriptions of the bait types, and coordinates of the field trials.

BCI.Opportunistic2018: Each row records one substrate sample that yielded Caenorhabditis nematodes during opportunistic sampling in 2018. The species found in each sample are indicated by $1 \mathrm{~s}$ in the relevant species columns.

LaSelva.Opportunistic2019: Each row records one Caenorhabditis isolate recovered during opportunistic sampling in 2019. Isolates identified by PCR from dried material on Whatman paper have names that start with FTA. Isolates identified from live cultures by mating tests have strain names that start with QG.

LaSelva.SamplesBySubstrate: Each row records one substrate sample that yielded Caenorhabditis nematodes during opportunistic sampling in 2019. The species found in each sample are indicated by $1 \mathrm{~s}$ in the relevant species columns.

\section{ACKNOWLEDGMENTS}

Work in the Barro Colorado Nature Monument was conducted under permits SEX/A-25-12 (2012), SEX/A-28-15 (2015), and SEX/A-55-18 (2018). We gratefully acknowledge the Republic of Panama and the staff of the Smithsonian Tropical Research Institute and Barro Colorado 
Island Research Station for their assistance. Work at La Selva Research Station was conducted under permits R-033, R-42, and R061-2019-OT-CONAGEBIO. We gratefully acknowledge the Republic of Costa Rica and the staff of the Organization for Tropical Research and La Selva Research Station for their assistance. We thank Christina Zakas and Sarah Rankin for help in the field, Andres Mansisidor, David Riccardi, Patrick Ammerman, Jia Shen, and Aurélien Richaud for help in the lab, and Marie-Anne Félix for helpful comments on the manuscript. This work was supported in part by NIH grants GM089972, GM121828, and GM141906 (MVR) and GM119744 (ABP).

\section{References}

Andrews, Simon. 2010. "FastQC: a quality control tool for high throughput sequence data." http://www.bioinformatics.babraham.ac.uk/projects/fastqc/

Baermann, G. 1917. "Ein Einfache Methode Zur Auffindung von Anklyostomum (Nematoden) Larven in Erdproben." Geneesk. Tijdschr. Nederlandsch-Indie 67: 131-137.

Baldi, Chris, Soochin Cho, and Ronald E. Ellis. 2009. "Mutations in two independent pathways are sufficient to create hermaphroditic nematodes." Science 326 (5955): 1002-5.

Bankevich, Anton, Sergey Nurk, Dmitry Antipov, Alexey A. Gurevich, Mikhail Dvorkin, Alexander S. Kulikov, Valery M. Lesin, et al. 2012. "SPAdes: a new genome assembly algorithm and its applications to single-cell sequencing." Journal of Computational Biology: A Journal of Computational Molecular Cell Biology 19 (5): 455-77.

Barrière, Antoine, and Marie Anne Félix. 2005. "High local genetic diversity and low outcrossing rate in Caenorhabditis elegans natural populations." Current Biology 15 (13): 1176-84.

Berg, Maureen, Xiao Ying Zhou, and Michael Shapira. 2016. "Host-specific functional significance of Caenorhabditis gut commensals." Frontiers in Microbiology 7 (OCT): 1-9.

Brůna, Tomáš, Katharina J. Hoff, Alexandre Lomsadze, Mario Stanke, and Mark Borodovsky. 2021. "BRAKER2: automatic eukaryotic genome annotation with GeneMark-EP+ and AUGUSTUS supported by a protein database." NAR Genomics and Bioinformatics 3 (1).

Buchfink, Benjamin, Chao Xie, and Daniel H. Huson. 2015. "Fast and sensitive protein alignment using DIAMOND." Nature Methods 12 (1): 59-60.

Capella-Gutiérrez, Salvador, José M. Silla-Martínez, and Toni Gabaldón. 2009. "TrimAl: A tool for automated alignment trimming in large-scale phylogenetic analyses." Bioinformatics 25 (15): 1972-73.

Camacho, Christiam, George Coulouris, Vahram Avagyan, Ning Ma, Jason Papadopoulos, Kevin Bealer, and Thomas L. Madden. 2009. "BLAST+: architecture and applications." BMC Bioinformatics 10: 421.

Chao, Anne, Nicholas J. Gotelli, T. C. Hsieh, Elizabeth L. Sander, K. H. Ma, Robert K. Colwell, and Aaron M. Ellison. 2014. "Rarefaction and extrapolation with hill numbers: a framework for sampling and estimation in species diversity studies." Ecological Monographs 84 (1): 45-67.

Chen, Shifu, Yanqing Zhou, Yaru Chen, and Jia Gu. 2018. "Fastp: an ultra-fast all-in-one fastq preprocessor." Bioinformatics 34 (17): i884-90.

Cook, Daniel E., Stefan Zdraljevic, Joshua P. Roberts, and Erik C. Andersen. 2017. "CeNDR, the Caenorhabditis elegans Natural Diversity Resource." Nucleic Acids Research 45 (D1): D650-57.

Crombie, Tim A., Stefan Zdraljevic, Daniel E. Cook, Robyn E. Tanny, Shannon C. Brady, Ye Wang, Kathryn S. Evans, et al. 2019. "Deep sampling of Hawaiian Caenorhabditis elegans reveals high genetic diversity and admixture with global populations." ELife 8: e50465.

Cutter, Asher D. 2015. "Caenorhabditis evolution in the wild." BioEssays 37: 983-95. 
Cutter, Asher D., Levi T. Morran, and Patrick C. Phillips. 2019. Males, outcrossing, and sexual selection in Caenorhabditis nematodes. Genetics 213: 27-57.

Dayi, Mehmet, Natsumi Kanzaki, Simo Sun, Tatsuya Ide, Ryusei Tanaka, Hayato Masuya, Kimiko Okabe, Hisashi Kajimura, and Taisei Kikuchi. 2021. "Additional description and genome analyses of Caenorhabditis auriculariae representing the basal lineage of genus Caenorhabditis." Scientific Reports 11: 1-15.

Dey, Alivia, Yong Jeon, Guo Xiu Wang, and Asher D. Cutter. 2012. "Global population genetic structure of Caenorhabditis remanei reveals incipient speciation." Genetics 191: 1257-69.

Dirksen, Philipp, Adrien Assié, Johannes Zimmermann, Fan Zhang, Adina-Malin Tietje, Sarah Arnaud Marsh, Marie-Anne Félix, et al. 2020. "CeMbio-The Caenorhabditis elegans Microbiome Resource" G3 10: 3025-39.

Dirksen, Philipp, Sarah Arnaud Marsh, Ines Braker, Nele Heitland, Sophia Wagner, Rania Nakad, Sebastian Mader, et al. 2016. "The native microbiome of the nematode Caenorhabditis elegans: Gateway to a new host-microbiome model.” BMC Biology 14: 38.

Dobin, Alexander, Carrie A. Davis, Felix Schlesinger, Jorg Drenkow, Chris Zaleski, Sonali Jha, Philippe Batut, Mark Chaisson, and Thomas R. Gingeras. 2013. "STAR: ultrafast universal RNA-seq aligner." Bioinformatics 29: 15-21.

Dubart, Maxime, Jelena H. Pantel, Jean Pierre Pointier, Philippe Jarne, and Patrice David. 2019. "Modeling competition, niche, and coexistence between an invasive and a native species in a two-species metapopulation." Ecology 100: 1-15.

Félix, Marie-Anne. 2021. WorldWideWorms. 2021. https://justbio.com/worldwideworms/.

Félix, Marie-Anne, Christian Braendle, and Asher D. Cutter. 2014. "A streamlined system for species diagnosis in Caenorhabditis (Nematoda: Rhabditidae) with name designations for 15 distinct biological species." PLoS ONE 9: e0118327.

Félix, Marie-Anne, Richard Jovelin, Céline Ferrari, Shery Han, Young Ran Cho, Eric C. Andersen, Asher D. Cutter, and Christian Braendle. 2013. Species richness, distribution and genetic diversity of Caenorhabditis nematodes in a remote tropical rainforest. BMC Ecology and Evolution 13: 10.

Félix, Marie-Anne, and Fabien Duveau. 2012. "population dynamics and habitat sharing of natural populations of Caenorhabditis elegans and C. briggsae." BMC Biology 10: 59.

Ferrari, Céline, Romain Salle, Nicolas Callemeyn-Torre, Richard Jovelin, Asher D. Cutter, and Christian Braendle. 2017. "Ephemeral-habitat colonization and neotropical species richness of Caenorhabditis nematodes." BMC Ecology 17: 1-13.

Frézal, Lise, and Marie Anne Félix. 2015. "C. elegans outside the petri dish." ELife 4: e05849.

Grabherr, Manfred G., Nir Brian J. Haas, Moran Yassour Joshua Z. Levin, Dawn A. Thompson, Ido Amit, Xian Adiconis, Lin Fan, Raktima Raychowdhury, Qiandong Zeng, Zehua Chen, Evan Mauceli, Nir Hacohen, Andreas Gnirke, Nicholas Rhind, Federica di Palma, Bruce W., and and Aviv Regev Friedman. 2013. "Trinity: reconstructing a full-length transcriptome without a genome from RNA-Seq data." Nature Biotechnology 29 (7): 644-52.

Green, Michael R., and Joseph Sambrook. 2020. "Total RNA extraction from Caenorhabditis elegans." Cold Spring Harbor Protocols 2020 (9): 101683.

Haas, Brian J., Alexie Papanicolaou, Moran Yassour, Manfred Grabherr, Philip D. Blood, Joshua Bowden, Matthew Brian Couger, et al. 2013. "De novo transcript sequence reconstruction from RNA-seq using the trinity platform for reference generation and analysis." Nature Protocols 8: 1494-1512.

Hamilton, W D. 1967. "Extraordinary sex ratios. a sex-ratio theory for sex linkage and inbreeding has new implications in cytogenetics and entomology." Science 156: 477-88.

Hill, Robin Cook, Carlos Egydio de Carvalho, John Salogiannis, Benjamin Schlager, Dave Pilgrim, and Eric S. Haag. 2006. "Genetic flexibility in the convergent evolution of hermaphroditism in Caenorhabditis nematodes." Developmental Cell 10 (4): 531-38.

Hoang, Diep Thi, Olga Chernomor, Arndt Von Haeseler, Bui Quang Minh, and Le Sy Vinh. 
2018. "UFBoot2: improving the ultrafast bootstrap approximation." Molecular Biology and Evolution 35: 518-22.

Hsieh, T. C., K. H. Ma, and Anne Chao. 2016. "INEXT: An R package for rarefaction and extrapolation of species diversity (Hill numbers)." Methods in Ecology and Evolution 7: $1451-56$.

Jurka, J., V. V. Kapitonov, A. Pavlicek, P. Klonowski, O. Kohany, and J. Walichiewicz. 2005. "Repbase update, a database of eukaryotic repetitive elements." Cytogenetic and Genome Research 110: 462-67.

Kanzaki, Natsumi, Isheng J. Tsai, Ryusei Tanaka, Vicky L. Hunt, Dang Liu, Kenji Tsuyama, Yasunobu Maeda, et al. 2018. "Biology and genome of a newly discovered sibling species of Caenorhabditis elegans." Nature Communications 9: 1-12.

Katoh, Kazutaka, and Daron M. Standley. 2013. "MAFFT multiple sequence alignment software version 7: improvements in performance and usability." Molecular Biology and Evolution 30: 772-80.

Kiontke, Karin. 1997. "Description of Rhabditis (Caenorhabditis) drosophilae n. sp. and R. (C.) sonorae n. sp. (Nematoda: Rhabditida) from saguaro cactus rot in Arizona." Fundamental and Applied Nematology 20: 305-15.

Laetsch, Dominik R., and Mark L. Blaxter. 2017. "BlobTools: interrogation of genome assemblies." F1000Research 6 (July). https://doi.org/10.12688/f1000research.12232.1.

Le, Si Quang, and Olivier Gascuel. 2008. "An improved general amino acid replacement matrix." Molecular Biology and Evolution 25 (7): 1307-20.

Leigh, Egbert G. 1999. Tropical Forest Ecology: A view from Barro Colorado Island. Oxford, Oxford University Press.

Letunic, Ivica, and Peer Bork. 2019. "Interactive Tree of Life (ITOL) v4: recent updates and new developments." Nucleic Acids Research 47 (W1): 256-59.

$\mathrm{Li}$, Heng. 2013. "Aligning sequence reads, clone sequences and assembly contigs with BWAMEM." arXiv [q-bio.GN]. arXiv. http://arxiv.org/abs/1303.3997.

Li, Shuning, Richard Jovelin, Toyoshi Yoshiga, Ryusei Tanaka, and Asher D. Cutter. 2014. "Specialist versus generalist life histories and nucleotide diversity in Caenorhabditis nematodes." Proceedings of the Royal Society B: Biological Sciences 281 (1777).

Lo, Yun-Hua, Yun Huang, Tho Son Le, Jung-Chen Hsu, Fang-Jung Yang, Tiffany Chang, Christian Braendle, and John Wang. 2021. "Prevalent sex ratio bias in Caenorhabditis nematodes. BioRxiv doi 10.1101/2021.10.26.465869.

Luca, Francesca De, Elena Fanelli, Monica Oreste, Gianluca Scarcia, Alberto Troccoli, Alessio Vovlas, Nicola Trisciuzzi, and Eustachio Tarasco. 2019. "Molecular profiling of nematode associates with Rhynchophorus ferrugineus in Southern Italy." Ecology and Evolution 9 (24): 14286-94.

Marek, Martin, Miloslav Zouhar, Ondrej Douda, Marie Manasová, and Pavel Ryšánek. 2014. "Exploitation of FTA cartridges for the sampling, long-term storage, and DNA-based analyses of plant-parasitic nematodes." Phytopathology 104 (3): 306-12.

McDade, Lucinda A., Kamaljit S. Bawa, Henry A. Hespenheide, and Gary S. Hartshorn. 1994. La Selva" Ecology and Natural History of a Neotropical Rain Forest. Chicago, University of Chicago Press.

Nguyen, Lam Tung, Heiko A. Schmidt, Arndt Von Haeseler, and Bui Quang Minh. 2015. “IQTREE: a fast and effective stochastic algorithm for estimating maximum-likelihood phylogenies." Molecular Biology and Evolution 32 (1): 268-74.

Noble, Luke M., John Yuen, Lewis Stevens, Nicolas Moya, Riaad Persaud, Marc Moscatelli, Jacqueline L. Jackson, et al. 2021. "Selfing is the safest sex for Caenorhabditis tropicalis." ELife 10: e62587.

Porazinka, Dorota L., Robin M. Giblin-Davis, Alejandro Esquivel, Thomas O. Powers , Wang Sung, and Thomas W. Kelly.(2010). Ecometagenetics confirms high tropical rainforest 
nematode diversity, Molecular Ecology 24: 5521-5530.

Osche, Gunther. 1952. Systematik und Phylogenie der Gattung Rhabditis (Nematoda). Zoologische Jahrbücher. Abteilung für Systematik, Ökologie und Geographie der Tiere 81(3): 190-280. Fischer Verlag, Jena, Germany.

Ren, Xiaoliang, Runsheng Li, Xiaolin Wei, Yu Bi, Vincy Wing Sze Ho, Qiutao Ding, Zhichao Xu, et al. 2018. "Genomic basis of recombination suppression in the hybrid between Caenorhabditis briggsae and C. nigoni." Nucleic Acids Research 46: 1295-1307.

Richaud, Aurélien; Zhang Gaotian; Lee, Daehan; Lee, Junho; Félix, Marie-Anne. 2018. "The local coexistence pattern of selfing genotypes in Caenorhabditis elegans natural metapopulations." Genetics 208: 807-21.

Schulenburg, Hinrich, and Marie Anne Félix. 2017. "The natural biotic environment of Caenorhabditis elegans." Genetics 206 (1): 55-86.

Seppey, Mathieu, Mosè Manni, and Evgeny M Zdobnov. 2019. "BUSCO: assessing genome assembly and annotation completeness." In Gene Prediction: Methods and Protocols, edited by Martin Kollmar, 227-45. New York, NY: Springer New York.

Simão, Felipe A., Robert M. Waterhouse, Panagiotis loannidis, Evgenia V. Kriventseva, and Evgeny M. Zdobnov. 2015. "BUSCO: assessing genome assembly and annotation completeness with single-copy orthologs." Bioinformatics 31: 3210-12.

Slatkin, Montgomery. 1977. "Gene flow and genetic drift in a species subject to frequent local extinctions." Theoretical Population Biology 12: 253-62.

Smit, Afa, and R. Hubley. 2010. "RepeatModeler Open-1.0." 2010. http://www.repeatmasker.org/RepeatModeler/.

Smit, Arian F. A., Robert Hubley, and P. Green. 1996. "RepeatMasker.” 1996. http://www.repeatmasker.org/.

Stevens, Lewis, Marie-Anne Félix, Toni Beltran, Christian Braendle, Carlos Caurcel, Sarah Fausett, David Fitch, et al. 2019. "Comparative genomics of 10 new Caenorhabditis species." Evolution Letters 3: 217-36.

Sudhaus, W, and K Kiontke. 2007. "Comparison of the Cryptic Nematode Species Caenorhabditis brenneri sp. n. and C. remanei (Nematoda: Rhabditidae) with the stem species pattern of the Caenorhabditis Elegans group." Zootaxa 1456: 45-62.

Sudhaus, Walter, Karin Kiontke, and Robin M. Giblin-Davis. 2011. "Description of Caenorhabditis angaria n. sp. (Nematoda: Rhabditidae), an associate of sugarcane and palm weevils (Coleoptera: Curculionidae)." Nematology 13: 61-78.

Theologidis, Ioannis, Ivo M. Chelo, Christine Goy, and Henrique Teotónio. 2014. "Reproductive assurance drives transitions to self-fertilization in experimental Caenorhabditis elegans." BMC Medicine 12: 93.

Wei, Qing, Yanmei Zhao, Yiqing Guo, Julie Stomel, Ryan Stires, and Ronald E. Ellis. 2014. "Cooption of alternate sperm activation programs in the evolution of self-fertile nematodes." Nature Communications 5: 5888.

Woodruff, Gavin C., Onyinyechi Eke, Scott E. Baird, Marie Anne Félix, and Eric S. Haag. 2010. "Insights into species divergence and the evolution of hermaphroditism from fertile interspecies hybrids of Caenorhabditis nematodes." Genetics 186: 997-1012.

Woodruff, Gavin C., and Patrick C. Phillips. 2018. "Field studies reveal a close relative of C. elegans thrives in the fresh figs of Ficus septica and disperses on its Ceratosolen pollinating wasps." BMC Ecology 18: 26.

Yang, Ziheng. 1994. "Maximum likelihood phylogenetic estimation from DNA sequences with variable rates over sites: approximate methods." Journal of Molecular Evolution 39: 30614.

Yoshiga, Toyoshi, Yuji Ishikawa, Ryusei Tanaka, Mantaro Hironaka, and Etsuko Okumura. 2013. "Species-specific and female host-biased ectophoresy in the roundworm Caenorhabditis japonica." Naturwissenschaften 100: 205-8. 
bioRxiv preprint doi: https://doi.org/10.1101/2022.01.13.476254; this version posted January 15,2022 . The copyright holder for this preprint (which was not certified by peer review) is the author/funder, who has granted bioRxiv a license to display the preprint in perpetuity. It is made available under aCC-BY 4.0 International license.

Zhang, Chao, Maryam Rabiee, Erfan Sayyari, and Siavash Mirarab. 2018. "ASTRAL-III: polynomial time species tree reconstruction from partially resolved gene trees." $B M C$ Bioinformatics 19 (Suppl 6): 15-30.

Zhang, Fan, Maureen Berg, Katja Dierking, Marie Anne Félix, Michael Shapira, Buck S. Samuel, and Hinrich Schulenburg. 2017. "Caenorhabditis elegans as a model for microbiome research.” Frontiers in Microbiology 8: 1-10. 